\title{
Adaptive Coding and Energy Efficient Packet Rate Transmission over Wireless LAN Friendly VOIP
}

\author{
Shruti Chaurasia \\ Department of computer science \\ Amity University sector -125 , \\ Noida (U.P.)
}

\author{
Kamal Kant \\ Department of computer science \\ Amity University sector -125, \\ Noida (U.P.)
}

\author{
Dr. Abhay Bansal \\ Department of Information \\ technology \\ Amity University sector -125 , \\ Noida (U.P.)
}

\begin{abstract}
Energy efficiency takes important in wireless communication systems where networked low-power devices relying on batteries should be able to communicate transparently. Current wireless network power management often substantially degrades performance and may increase overall energy usage when used with latency-sensitive applications. We propose power management that adapts its behaviour to the access patterns and intent of applications, the characteristics of the network interface, and the energy usage of the platform. We have implemented software for power management as a windows family module--our results show substantial benefits for distributed file systems and thin-client applications. Compared to default $802.11 \mathrm{~b}$ power management, STPM reduces the total energy usage of an PPAQ running the Coda distributed file system by $21 \%$ while also reducing interactive file system delay by $78 \%$. Further, STPM adapts to diverse operating conditions: it yields good results on both laptops and handhelds, supports $802.11 \mathrm{~b}$ network interfaces with substantially different characteristics, and performs well across a range of application network access patterns.
\end{abstract}

Keywords:-VoIP (voice over internet protocol),radio frequency, Fixed-Mobile Convergence (FMC), Power Save Mode (PSM), Automatic Power Save Delivery (APSD), Access point (AP), Session Initiation Protocol (SIP), Traffic Indication Map(TIM).

\section{INTRODUCTION}

Many public IEEE802.11 wireless networks have been deployed not only in building but also in parks and streets, the importance of Voice over IP (VoIP) over wireless networks has been increasing, encouraging efforts to improve Quality of Service and increase the energy efficient packet transmission for VOIP.[1], [2], [3]. Dual mode phones are the emerging trend for cellular and wireless LAN (WLAN). Beside from data access, the WLAN can also be leveraged for packet transmission in Voice over Internet Protocol (VoIP) calls. Traditional calling over the cellular interface have two advantages.

1) Calls over the Internet through WLANs are cost effective.

2) Packet rate transmission is not affected by lack of coverage of the cellular network in indoor areas or outdoor area.

Energy consumption over WLAN has greater concern. Active or even idle wireless network interface (WNIC) is a significant drain on the relatively limited capacity batteries Found in smart phones. For example, the specifications of Apple's iPhone [5] lists a talk time of 14 hours with the cellular interface, but no more than 6 hours of operating Life time with the WLAN interface for very light web browsing and e-mail access The D-Link VClick dual mode- smart phone lists a talk time of 5 hours with the cellular interface, and only 2 hours of operating lifetime with the WLAN interface [8]. Previous studies in energy efficient packet transmission gives result that for high end devices e.g. laptops, at least 15-20\%of the total energy capacity is consumed by an active WLAN interface and for low-end devices e.g. PDA, this increases to about 65 percent of total energy consumption.

Packet generation intervals of the order of tens of milliseconds exacerbate the situation by making it difficult for the radio to spend any significant amount of time in the sleep mode.

In this paper, we address the issue of reducing energy consumption of the WLAN interface during a packet transmission over VoIP and preserving the quality in acceptable levels. Our approach is based on an green call algorithm that can be implemented as a software solution to saving energy. This software solution work with legacy interfaces whose radios are not be as power efficient as those of emerging interfaces.

\section{POWER SAVING AND DEL REDUCTION FOR WLAN FMC SERVICES}

Turning off Radio Frequency (RF) transceiver is an efficient method for prolonging a battery life-time of a mobile station in wireless networks, but it has a trade-off relationship with real-time traffic delay like Voice over Internet Protocol (VoIP). This paper presents a powerefficient packet rate transmission based on adaptive sleep threshold adjustment in mobile phones with WLANbased Fixed-Mobile Convergence (FMC) service. The basic idea is that the sleep threshold that establishes how long a Radio Frequency transceiver of a terminal stays in a low power state is determined adaptively and adjusted according to network traffic in service the terminal systems are implemented and evaluated by measuring the energy efficient power

consumption. The experimental results show that the proposed approach can minimize power consumption. 
Smartphone's such as Apple's iPhone, Nokia's N95, and Samsung's Galaxy has accelerated the usage of WLANbased Fixed-Mobile Convergence (FMC) services more rapidly. Such types of smart phones are in demand of consumer electronics be successful, it have a long battery life-time while maintaining a small form factor like a traditional single-mode cellular phone IEEE 802.11 Power Save Mode (PSM) was specified power reduction in a mobile terminal with WLAN network card.

Non-real time data e.g. e-mail, the 802.11 PSM scheme is a useful mechanism for reducing power consumption effectively. There are some limitations when these scenarios are used for voice communication in which the traffic is composed of delay-sensitive data like voice or call signaling. It becomes a major factor for the large packet delay between terminal and access point (AP) in a situation where transmission from sender does not occur in a substantial delay between two outgoing packets. Researches [8][9][10] related to the 802.11 PSM operation has focused on the extent to which energy efficient power consumption in data rate transmission can be reduced by controlling the sleep threshold. The existing approaches [11] do not support network-adaptive solution.

Automatic Power Save Delivery (APSD) is the newest method specified in the 802.11e standard to improve Quality of Service. If we utilize this mechanism it is necessary to replace all APs already installed worldwide new ones adopting the 802.11 amendment in order to overcome the above limitations.

The problems arise from the 802.11 PSM operations when user communicates with WLAN in an FMC terminal .This problem occurs when a station cannot receive the buffered downlink frame in a situation where the transmission from sender does not occur or there is a delay between two outgoing packets, a receive request frame is synchronized with the transmission packet categorized into two cases: delay during call signaling and delay during talk time. Delay during call signaling is occurred when a receiving station stays in sleep state for incoming signaling.

The following call procedure between phone A and B using the [12] Session Initiation Protocol (SIP) protocol as illustrated in Fig.1.

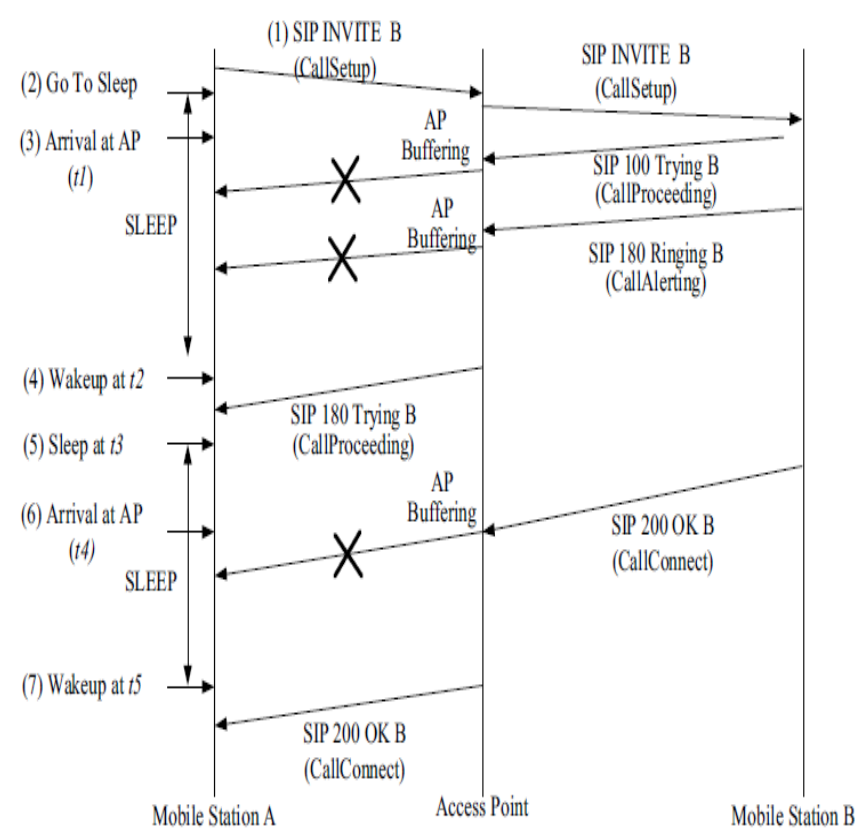

Fig. 1.Delay during Call Signaling

The delay during call signaling depicted in Fig.1 may be described as follows:

1. User A tries to make a call to B. The Call Manager (CM) of A sends "SIP INVITE B".

2. The WLAN subsystem of user A goes to sleep immediately for $1500(\mathrm{~ms})$.

3. User B is not busy. It sends reply message "SIP 180 ringing".

4. The "SIP 180 Ringing" message from user B will arrive and be buffered at access point.

5. User A wakes up after $1500(\mathrm{~ms})$ at time $\mathrm{t} 2$. It will investigate the Traffic Indication Map (TIM) frame and find some data at the access point. After that terminal send a Power Save-Poll (PS-Poll) frame and take buffering data SIP 180 Ringing at time $\mathrm{t} 2$.

6. User A goes to sleep after some time (no data to send).User B hooks off a ringing phone will send a "SIP 200 OK" message to A. 

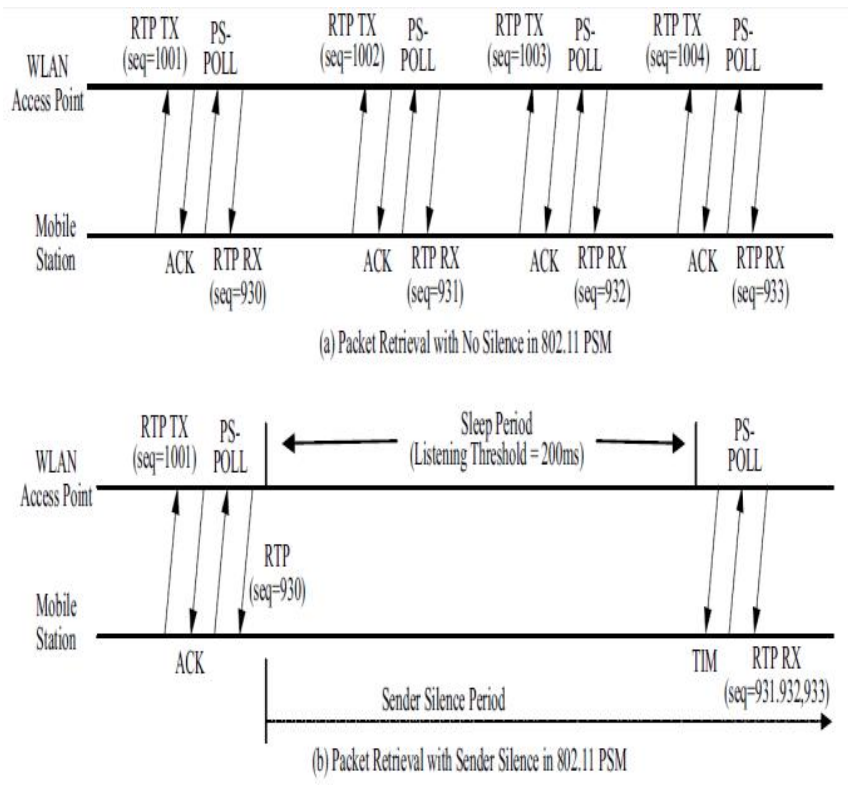

Fig. 2.Packet arrival patterns with and without
Sender Silence in 802.11 PSM

Voice packets from peer are being retrieved from AP synchronized with transmitted packets interval so, delay between consecutive packets will not be increased except for network condition degradation.

The waiting (sleep) time before retrieving next data packet is at least $100(\mathrm{~ms})$ for silence period. The burst packets retrieval after receiving Traffic Indication Map (TIM) increases jitter variation at voice reception block, which degrades voice quality.802.11 PSM efficiently works when it is used for real-time traffic periodically generated both in uplink and downlink. Using PSM to save energy during a VOIP call the two ends of a VoIP call are peers of each other. The device on the other end of the client will be referred to as the peer. If both ends are running an energy saving algorithm, they are the client and other as the peer. If the client uses PSM to go to sleep, any packets arriving from the peer will be buffered at the AP.

If the arriving packets were delay-tolerant, the client could sleep for long durations before collecting packets from the AP. VoIP traffic has small tolerable latencies and each packet must reach the client by its play out deadline. That's why client sleep schedules must be precise enough to ensure no packets are lost due to missed play out deadlines.

To calculate such a strict sleep and wake-up schedule, we need to consider the latency (mouth-to-ear delay) of a packet from the peer to the client.

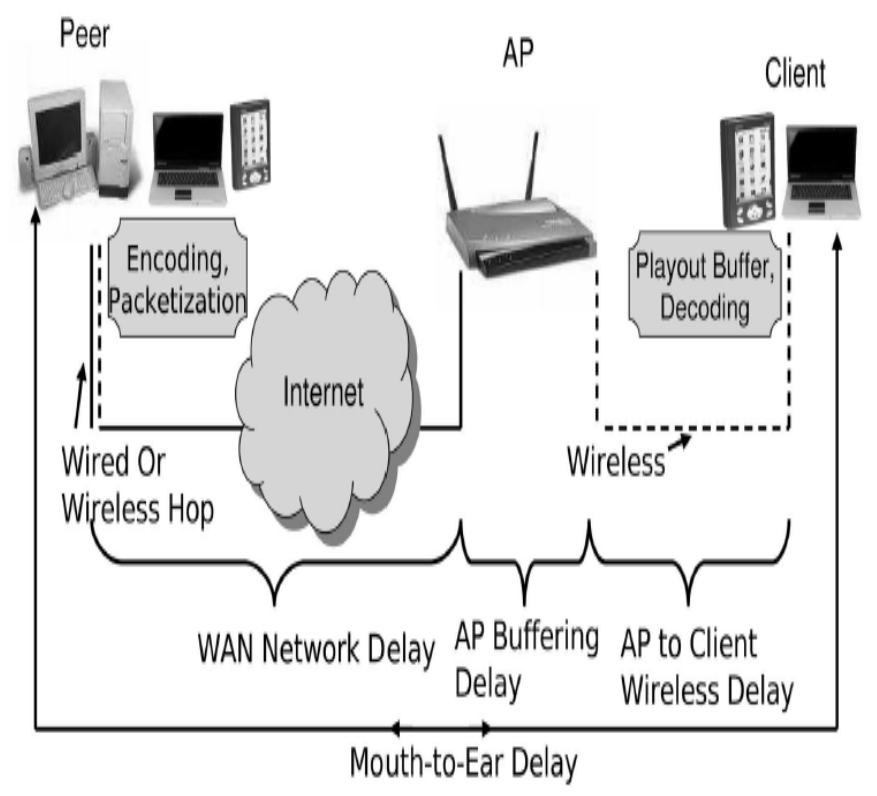

Fig. 3.Illustration of end-to-end Latency omponents of a VoIP call between a Peer on a Wired/Wireless Network and the Client on a Wireless Network.

The latency from the peer to client's AP is mainly the network delay for the packet once is sent from the application layer of the peer station. The minimum latency induced by the play out buffer is only time to decode and play out the packet. When the mouth-to-ear delay exceeds a specific tolerable value the packet is dropped.

\section{GREEN CALL ALGORITHM}

To calculate sleep periods, the complete Green Call algorithm derive sleep schedules during the call or packet transmission. Green- Call handles unknown network latencies by keeping track of latencies which is suffered by previous packets received at client. Predicts latency bounds for future network latencies, based on subsequent sleep periods are calculated the magnitude of shift value $\mathrm{S}$ used for network latency bound depends on the current loss rate. at higher loss rates, more conservative sleep periods are used. This enables a smooth trade-off between loss rate and energy savings and is the main feature of the algorithm.

The Green Call pseudo code is presented as an algorithm in Fig. 4. 


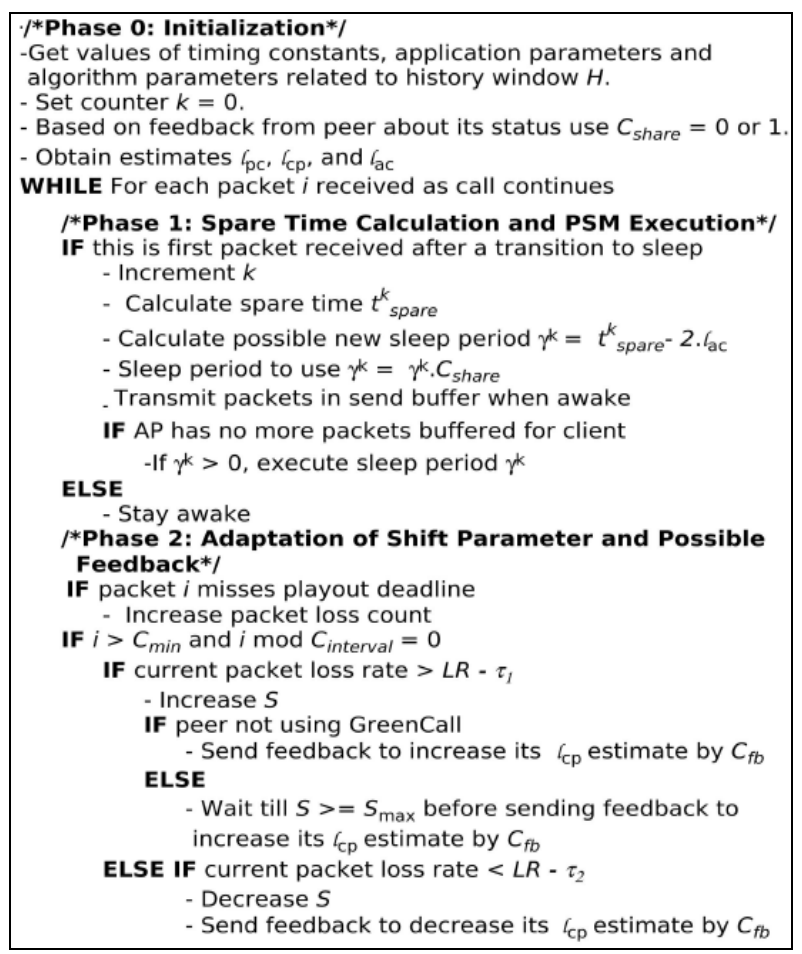

\section{Fig.4 Green call Algorithm}

The algorithm can be divided into three phases:

An initialization phase followed by two phases as each Packet arrives.

\subsection{Phase 0}

It deals with the collection of parameters defined by the application as well as tunable parameters of the algorithm. The final step of this phase is to estimate the one way first packet network latencies lpc and lcp between the client and the peer and vice versa, and the one way latency between client and AP, lac. This is done by sending ICMP echo packets from the client to each of these points to get the RTT.

\subsection{Phase 1}

It begins with the calculation of spare time for packets as the algorithm loops for each packet receive until the call continues. Calculation is work only for the first packet after a sleep period it signifies a decision point of the algorithm from where the next sleep period is derived. Once the Access Point has no packets for buffer client goes to sleep for duration $\mathrm{k}$ duration of kith sleep period. Duration k considers whether the peer is running Green Call. When the sleep period is not greater than zero, the client just stays in the constantly awake mode (CAM). To check that client is not interrupt its sleep period to send packets, to the client buffers generated packets until it wakes up. In wakeup mode the client contends for the medium with downlink packets from AP to send its packets.

\subsection{Phase 2}

It deals with the adaptation of S. Large values of S will result in more conservative sleep periods it minimizing packet losses. Network losses are tolerable we can save more energy by being more aggressive in selecting a sleep period with smaller values of S. To stay with a target loss rate LR we achieve maximum possible energy savings.

The monitoring begins after minimum numbers of packets, Cmin have been received, and it is done every $\mathrm{C}$ interval packets and thresholds used to control loss rate below LR. If the peer is running energy saving algorithm client tries to control its loss rate through adaptation of S. Once the maximum $\mathrm{S}$ has been reached it sends a feedback to the peer for it to increase estimate of lcp such that future sleep periods take into account. An increased lcp would decrease sleep times at peer and improve loss rate situation at client. Once loss rate is back below LR 2 client sends feedback again to peer decrease its lcp to original levels.

The adaptation of $\mathrm{S}$ is done through two constant factors: Cincf to increase it and Cdecf to decrease Green Call operation without feedback. It is desirable that the client runs independently from the peer without any mutual feedback the peer might be running on a device on which adding new software (to run Green Call) is not feasible or desirable

\begin{tabular}{|l|c|c|}
\hline & Energy (mWh) & \% Savings \\
\hline GreenCall & 2142 & \multirow{2}{*}{20.9} \\
\hline No GreenCall & 2707 & \\
\hline
\end{tabular}

Fig.5 Energy Savings in a Day with and without Green Call during Calls

\section{RESULT and Discussion}

The results obtained by running Green Call under adaptive packet rate transmission over wireless LAN over friendly VOIP. We also examine the effect of asymmetric network paths on the play out deadline estimation accuracy of Green Call. This paper provides insight on the expected energy savings with Green Call. We will use a packet generation interval of $60 \mathrm{~ms}$ and had the peer also running Green Call. The results are shown in Figures

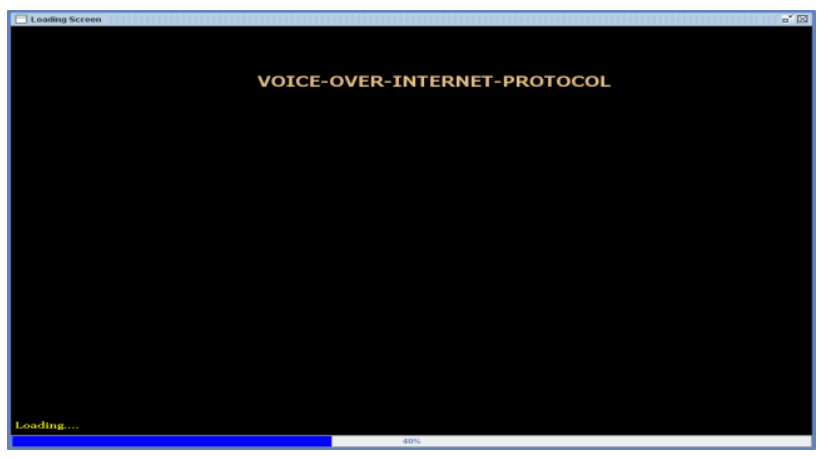

Fig.6.1. Loading of VOIP protocol 


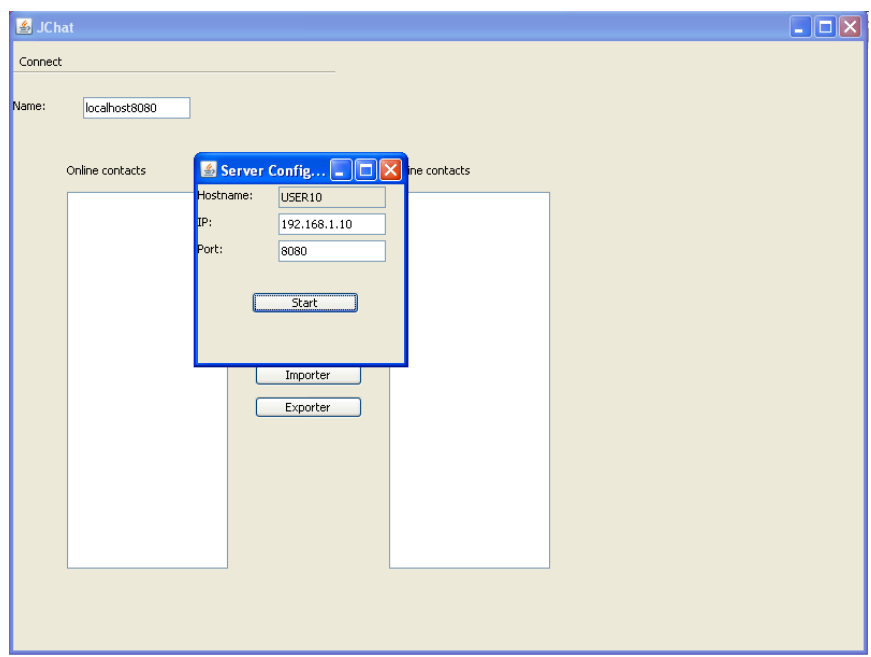

Fig. 6.2 VOIP Server is started

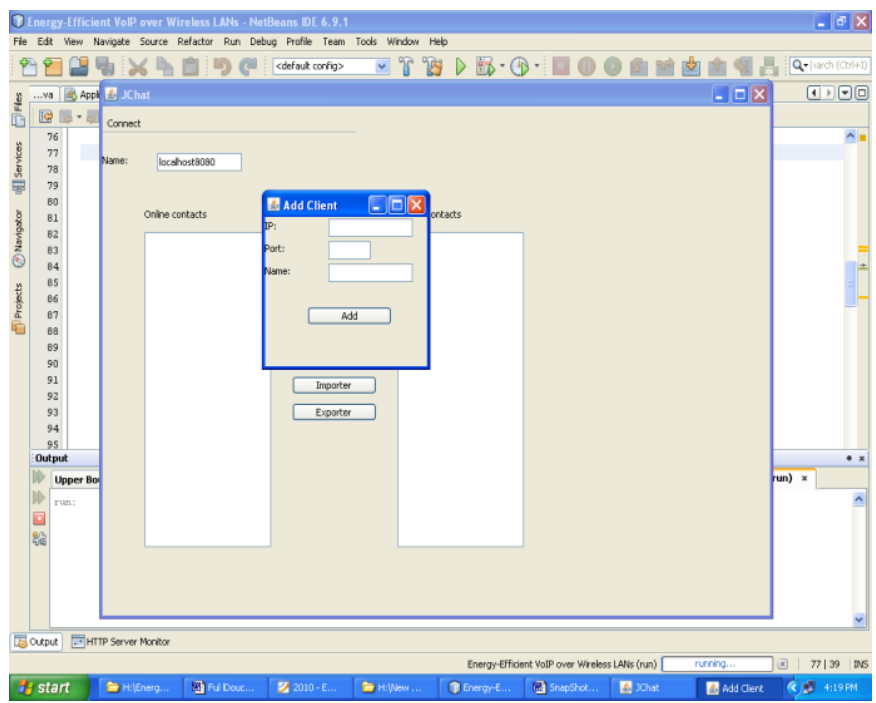

Fig. 6.3 Adding Online Client to VOIP

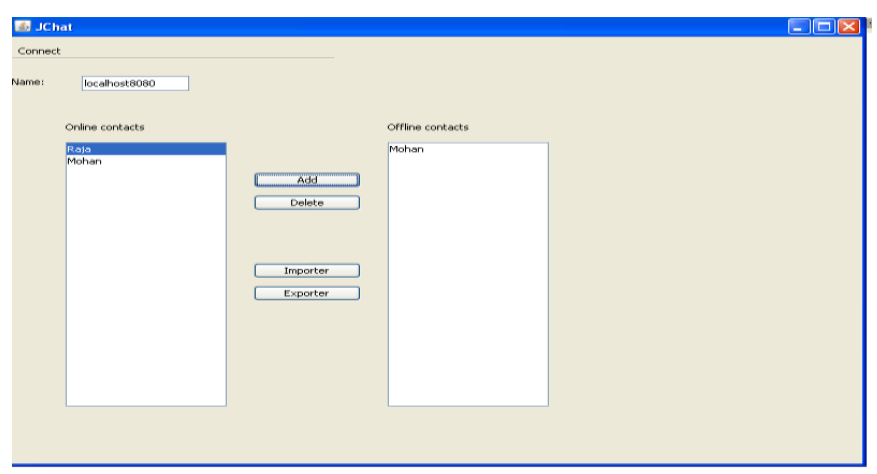

Fig. 6.4 Adding Offline Client to VOIP

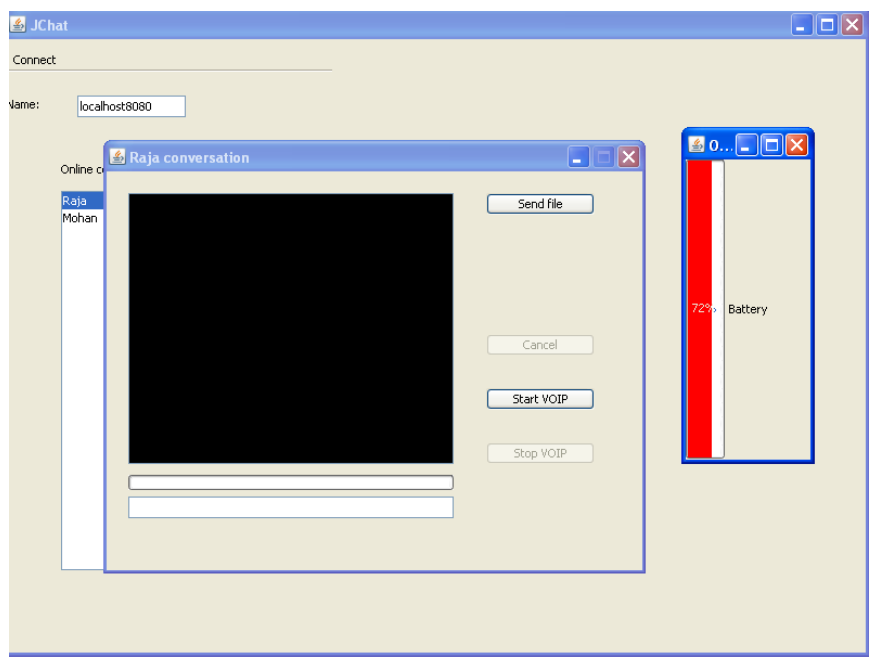

Fig. 6.5 Showing Energy Consummation by Client

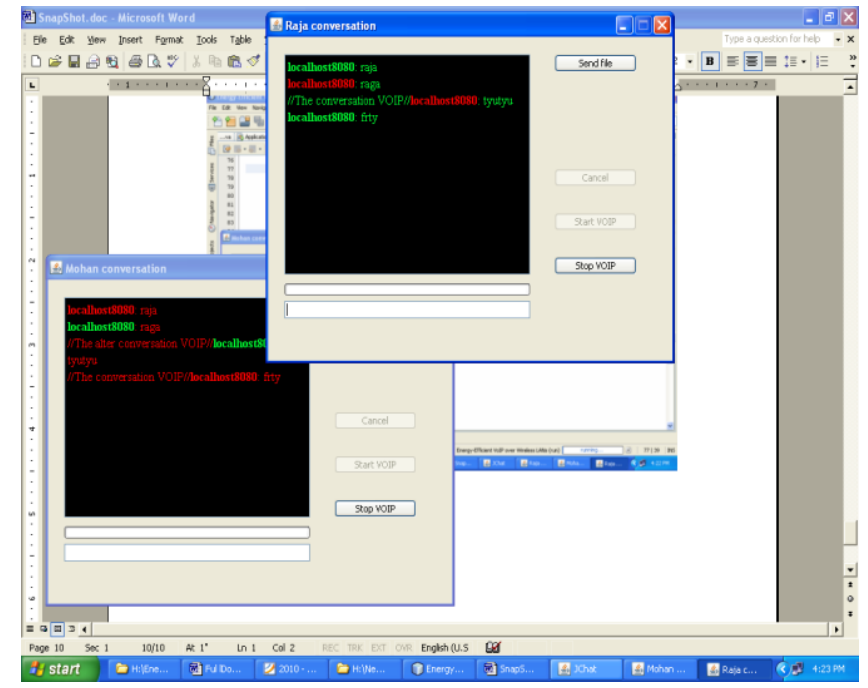

Fig. 6.6 Packet Rate Transmission

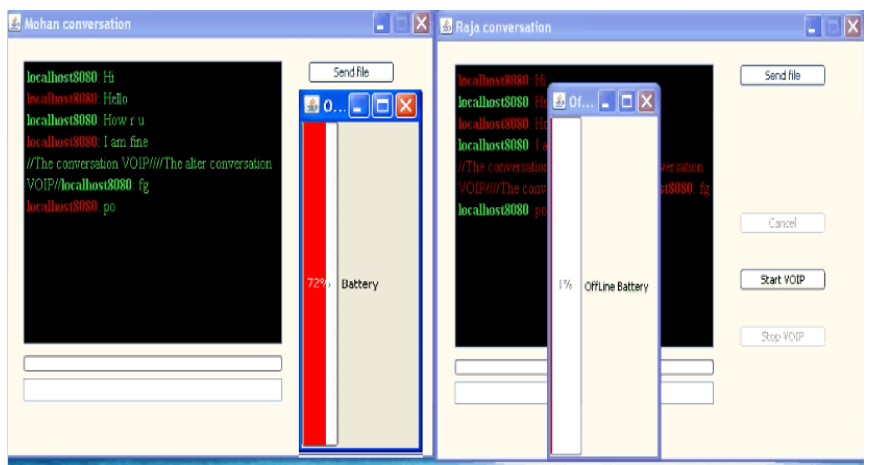

Fig 6.7 Show Energy Consumption in Awake and Sleep Mode

The result for the typical scenario shows that greater than two-thirds of the energy consumed by the WLAN interface can be generally saved to different geographic points while keeping the packet loss rates down to tolerable levels as specified. It can be seen that the energy savings are done at sleep and a wake mode of packet rate transmission in VoIP. 


\section{CONCLUSION}

This work is to improve the Quality of Service (QoS) of voice over internet protocol by proposing software for consuming energy over WLAN reducing the delay during the power save operation. Energy consumption is very important factor determining whether a mobile phone will be successful. Aim of saving energy by delaying and early dropping packets with respect to target delay and packet loss constraints. It improves performance by transmitting aggregated packets at their group rate, which has effect of reducing air-time.

\section{REFERENCES}

[1] D.P. Hole and F.A. Tobagi, "Capacity of an IEEE 802.11b WirelessLAN Supporting VoIP," Proc. IEEE Int'l Conf. Comm. (ICC '04),pp. 196-201, 2004.

[2 M. Veeraraghavan, N. Cocker, and T. Moors, "Support of VoiceServices in IEEE 802.11 Wireless LANs," Proc. IEEE INFOCOM,vol. 1, pp. 488-497, 2001.

[3] D. Chen, S. Garg, M. Kappes, and K.S. Trivedi, "Supporting VoIPTraffic in IEEE 802.11 WLAN with Enhanced Medium AccessControl (MAC) for Quality of Service," technical report, AvayaLabs Research, 2002.

[4] S. Garg and M. Kappes, "An Experimental Study of Throughput for UDP and VoIP Traffic in IEEE 802.11b Networks," Proc. IEEE Wireless Comm. and Networking Conf. (WCNC '03), vol. 3, pp. 1748- 1753, 2003.

[5] "Wi-Fi Phones Make a Splash," CNET News, http://news.com/

$\mathrm{Wi}$ -
[6] "New T-Mobile Plan Cuts Costs by Using Internet," San FranciscoChronicle, http://sfgate.com/cgibin/article.cgi?f=/c/a/2007/06/27/BUGG6QM5DR1. DTL, 2009.

[7] "Apple Iphone Technical Specifications," http://www.apple.com/iphone/specs.html, 2009.

[8] "D-Link V-CLICK Makes It Easy to Switch from GSM and Wi-FiModes with a Single Click," http://www.slashphone.com/115/5464.html, 2009.

[9] R.Krashinsky, and H. Balakrishnan, "Minimizing energy for wireless web access with bounded slowdown ", ACM Wireless Networks, Vol.11, No.1, pp.135-148, 2005.

[10] R. Banginwar, and E. Gorbatov, "Gibraltar: Application and Network Aware Adaptive Power Management for 802.11", In Proc. of the 2005 Second Annual Conference on Wireless On-demand Network Systems and Services, pp.98-108,January 2005.

[11] T. Shiao-Li, and C. E-Cheng, "Reducing idle mode power consumption of cellular/VoWLAN dual mode mobiles", In Proc. of the 2005 IEEE Global Conference on Telecommunications, pp.2902-2906, December 2005.

[12] Internet Engineering Task Force Network Working Group, J. Rosenberg, H. Schulzrinne, G. Camarillo, A. Johnston, J. Peterson, R. Sparks, M.Handley, and E. Schooler, "SIP: Session Initiation Protocol", $R F C$ 3261,June 2002. 5296745.html, 2009 